\title{
Drop evaporation on rough hot-spots: effect of wetting modes
}

Huacheng Zhang ${ }^{1}$, Yutaku Kita ${ }^{1}$, Dejian Zhang', Gyoko Nagayama ${ }^{2}$, Yasuyuki Takata, ${ }^{1,3}$, Khellil Sefiane ${ }^{4}$, Alexandros Askounis ${ }^{3,5 *}$

${ }^{1}$ Department of Mechanical Engineering, Graduate School of Engineering, Kyushu University, 744 Motooka, Nishi-ku, Fukuoka 819-0395, Japan

${ }^{2}$ Department of Mechanical Engineering, Kyushu Institute of Technology, 1-1 Sensui, Tobata, Kitakyushu, Fukuoka 804-8550, Japan

${ }^{3}$ International Institute for Carbon-Neutral Energy Research (WPI-I2CNER), Kyushu University, 744 Motooka, Nishi-ku, Fukuoka 819-0395, Japan

${ }^{4}$ Institute for Multiscale Thermofluids, School of Engineering, The University of Edinburgh, King's Buildings, Robert Stevenson Road, Edinburgh EH9 3FB, United Kingdom

${ }^{5}$ Engineering, Faculty of Science, University of East Anglia, Norwich Research Park, Norwich, NR4 7TJ, United Kingdom

*To whom correspondence should be addressed: E-mail: a.askounis@i2cner.kyushu-u.ac.jp; Tel.: +81-92-802-3905 Fax: +81-92-802-3905.

\section{Abstract}

Hot-spots are a common occurrence in power electronics which become increasingly hotter as chips become denser. Novel cooling technologies are emerging to cope with this increasing heat load, which imbed a condenser to supply cooling drops to the hotspots. Nonetheless, the evaporation process of the drops has been overlooked. Here, we conducted a series of experiments to understand how the evaporation and motion of drops are influenced by the wetting mode of rough hot-spot. We fabricated three different surfaces exhibiting full (Wenzel) or partial (Cassie-Baxter) wetting and the hot-spot is imposed by laser irradiation. We report a direct link between drop motion and wetting mode with the partial wetting drops being highly mobile, attributable to the 
lowest pinning energy and interpret based on an energy analysis. This work provides a framework for future modifications in hot-spot cooling to account for drop motion which should greatly influence the overall heat removal performance.

\section{Introduction}

Electronic microchips are becoming more densely integrated due to miniaturization. However, increasing the density of the microchips has resulted in greater local generation of heat, also known as hot-spots, that cannot be removed with conventional air-cooling methods[1,2]. Drop evaporation is capable of removing large amounts of heat and has been proposed in emerging hot-spot cooling technologies[3, 4]. However, the evaporation process of drops on hot-spots is far from understood as it is a complex problem combining the non-trivial issues of evaporation dynamics and wetting of drops which may be influenced by parameters such as surface softness[5] or surface roughness[6] with the heating power effect of the hot-spots[7]. In what follows, we will mainly focus on the role of surface roughness on the evaporation process and the contact line (CL) motion kinetics of drops on rough, hydrophobic hot-spots.

Rough surfaces can be fabricated by patterning a surface with either periodic, e.g. micropillars[8], or random[9] topological characteristics, resulting in tuneable surface adhesion properties. Surface adhesion infers to the ability of the surface to hinder the motion of the drops and mainly depends on how the roughness features are wetted. When the liquid fully penetrates the roughness features, the CL has to overcome the 
energy barrier imposed by the features rendering the surface "sticky", as described by Wenzel[10]. On the other hand, should the liquid partially wet the surface asperities, then the surface features impose a very small energy barrier rendering the surface "slippery", as described by Cassie-Baxter[11]. Great scientific effort has focused on the fabrication[9, 12, 13] and the wetting properties of these surfaces[14-17].

The relation between wetting properties of rough surfaces and the natural evaporation kinetics of liquid drops is considerably understood[17-21]. However, the influence of heating these surfaces on drop evaporation kinetics remains elusive, with only a limited number of works[22-25]. This lack of information becomes increasingly important in emerging cooling technologies, where a specially designed condenser imbedded in an integrated cooling system constantly provides drops to the hot-spot via coalescence-induced jumping drops [3, 4].

In this contribution, we investigate how the kinetics of evaporation and CL motion of water drops on rough hot-spots are influenced by the wetting mode of the surface. In particular, we fabricated a series of surfaces with varying roughness which resulted in distinct wetting regimes: partial or full wetting. To uncouple the relation between wetting and evaporation kinetics on hot-spots we used a combination of an optical camera to record the change in the shape of the drops from the side and an infrared camera from the top to follow the CL motion with high contrast. We report strong pinning in the full wetting cases which highly deforms the CL and mobile drops in the case of partial wetting. Energy analysis of each drop provided the necessary information on the underlying pinning mechanism. Our results will provide the necessary 
information on the overlooked part of evaporation in designing highly efficient hot-spot cooling technologies.

\section{Experimental setup}

\section{Surface fabrication}

In this study, pure aluminium surfaces (Al 1100, $20 \mathrm{~mm} \times 20 \mathrm{~mm} \times 0.5 \mathrm{~mm}$ ) were used with initial surface roughness of $0.254 \pm 0.039 \mu \mathrm{m}$. The surfaces were polished with sandpaper $(320,600,1000$, FUJI STAR) and cleaned in an ultrasonic bath of acetone and ethanol solutions for 10 minutes separately and rinsed with deionized (DI) water. The clean substrates were etched by anodizing them at a constant voltage of 20 $\mathrm{V}$ in $8 \% \mathrm{H}_{2} \mathrm{SO}_{4}$ solution at room temperature for 2 hours, 4 hours and 6 hours respectively to obtain different surface roughness. After etching, each surface was rinsed with DI water, dried under a stream of compressed air for 20 seconds and was subsequently sprayed with water-repellent fluoro-resin and left to dry at room temperature for more than 12 hours. 


\section{Surface characterization}
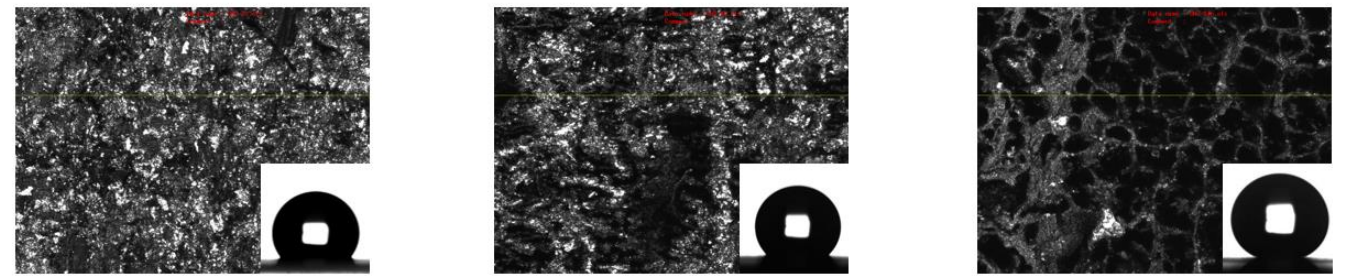

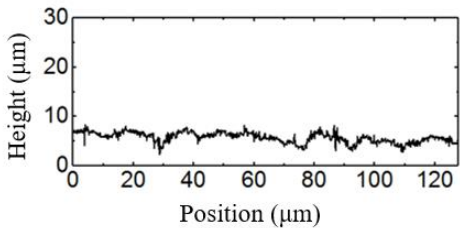

(a)

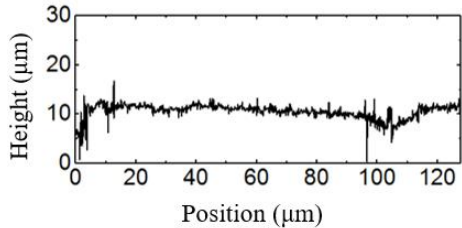

(b)

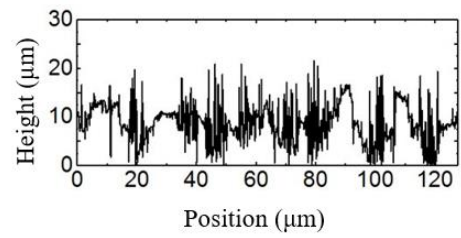

(c)

Figure 1. Top row shows CLSM images of the surfaces etched for (a) 2 hours, (b) 4 hours and (c) 6 hours. Insets are optical micrographs of water drops depicting the contact angles on each surface. Bottom row depicts the corresponding cross-sectional profiles of each surface.

A confocal laser scanning microscopy (CLSM, Olympus-LEXT OLS 3000) was employed to study the morphological features of the surfaces. CLSM micrographs of the test surfaces are shown in Figure 1. Insets show the shape of $10 \mu 1$ of DI water drops on each surface, captured by a charge-coupled device (CCD) camera. Surface characterization information is summarized in Table. 1. Surfaces are labelled as: $\mathrm{S}$ referring to surface and $2 \mathrm{~h}, 4 \mathrm{~h}, 6 \mathrm{~h}$ representing the etching time. Longer etching time, results in rougher surfaces and higher contact angles, as reported previously[26, 27]. 
Table. 1 Etch time, contact angle and surface roughness for different samples.

\begin{tabular}{|c|c|c|c|c|c|c|}
\hline $\begin{array}{c}\text { Hot- } \\
\text { spot } \\
\text { name }\end{array}$ & $\begin{array}{c}\text { Etching } \\
\text { time } \\
\text { (hours) }\end{array}$ & $\begin{array}{c}\text { Surface } \\
\text { roughness, } \\
\mathrm{R}_{\mathrm{a}}(\mu \mathrm{m})\end{array}$ & $\begin{array}{c}\text { Apparent } \\
\text { contact } \\
\text { angle, } \\
\theta_{a}(\mathrm{deg})\end{array}$ & $\begin{array}{c}\text { Advancing } \\
\text { angle, } \\
\theta_{\text {adv }} \\
(\mathrm{deg})\end{array}$ & $\begin{array}{c}\text { Receding } \\
\text { angle, } \\
\theta_{\text {rec }} \\
(\mathrm{deg})\end{array}$ & $\begin{array}{c}\text { Contact } \\
\text { angle } \\
\text { hysteresis, } \\
\text { CAH (deg) }\end{array}$ \\
\hline $\mathrm{S}-2 \mathrm{hr}$ & 2 & $\begin{array}{c}0.719 \pm \\
0.038\end{array}$ & $124 \pm 3$ & $132 \pm 4$ & $91 \pm 3$ & $41 \pm 2$ \\
\hline $\mathrm{S}-4 \mathrm{hr}$ & 4 & $\begin{array}{c}1.114 \pm \\
0.038\end{array}$ & $130 \pm 3$ & $141 \pm 2$ & $109 \pm 3$ & $32 \pm 3$ \\
\hline $\mathrm{S}-6 \mathrm{hr}$ & 6 & $\begin{array}{c}2.761 \pm \\
0.038\end{array}$ & $150 \pm 3$ & $154 \pm 3$ & $137 \pm 2$ & $17 \pm 2$ \\
\hline
\end{tabular}

\section{Evaporation on hot-spots experiments}

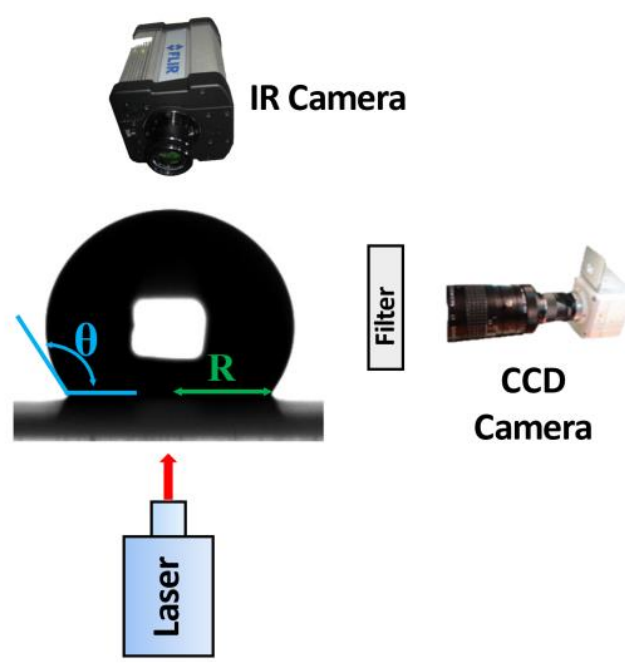

Figure 2. Schematic illustration of the experimental setup.

The experimental setup is shown in Figure 2. In particular, $10 \mu \mathrm{L}$ DI water drops were gently deposited on the surfaces with a micropipette, for accurate dosage. The evolution of drop shape (apparent contact angle, $\theta$, and radius, $R$ ) was followed by a Sentech STC-MC152USB CCD camera from the side. To precisely follow the motion of the CL, we placed an FLIR SC4000 mid-infrared (IR) camera (spectral range from 3.0 to $5.0 \mu \mathrm{m}$, resolution of $18 \mathrm{mK}$ ) above the drop, which provided high contrast between water and substrate. Hot-spots of constant power were imposed by heating each substrate from below with an Integra-MP-3022 diode laser (808 nm wavelength, 
Spectra-Physics), operated under continuous wave mode at $1.9 \mathrm{~W}$ as measured by a laser meter (Vega, Ophir Optronics Solutions Ltd.). Exemplary infrared images of each hot-spot and the approximate position of the drops are shown in Figure 3. Each experiment was conducted 10 times for reproducibility, inside an environmental chamber to control temperature and relative humidity at $18.0 \pm 1.0^{\circ} \mathrm{C}$ and $40 \pm 10 \%$.
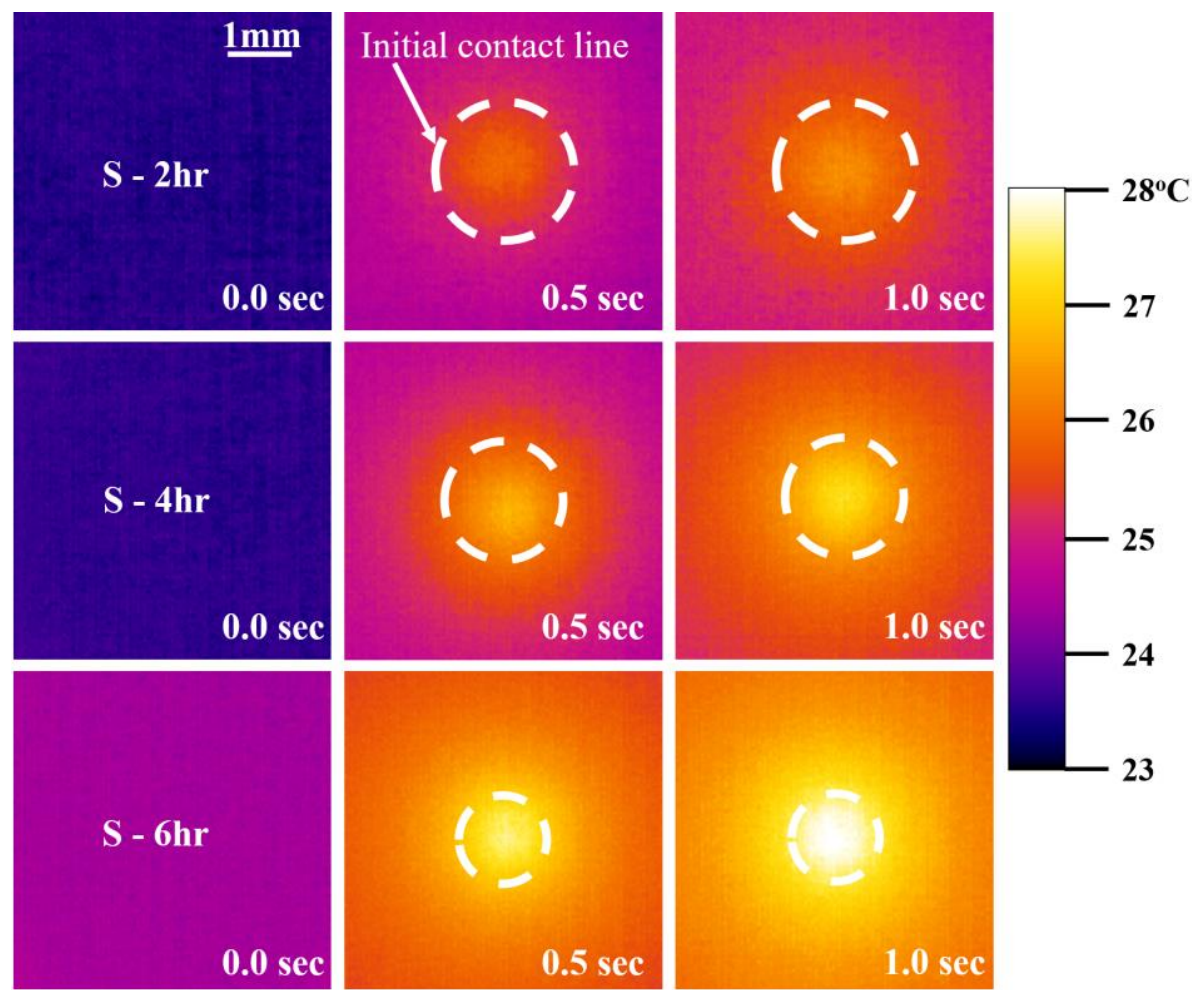

Figure 3: Infrared micrographs of each hot-spot used in this study. Dashed lines correspond to the initial contact line.

\section{Result and discussion}

\section{Drop evaporation}

Initially, we conducted evaporation experiments on each surface fabricated without heating which should act as a reference point to better understand the influence of the hot-spots on the evaporation process and CL motion kinetics. Figure 4 shows the 
evolution of the contact radius and the contact angle as a function of time $(t)$ normalised by drop lifetime $\left(t_{0}\right)$ i.e. $t / t_{0}$, of water drops on the non-heated surfaces with increasing roughness from top to bottom. In the smoothest ( $\mathrm{S}-2 \mathrm{hr}$ ) case, the drop evaporates under the constant contact radius (CCR) regime with decreasing contact angle until ca. $75 \%$ of its lifetime as the dashed line shown in Figure 4. As the contact angle reaches about 65 degrees, the evaporative behaviour shifts to constant contact angle (CCA) regime which continues until full evaporation. The drop on the (S-4hr) case evaporated under CCR regime for ca. $60 \%$ of its lifetime as indicated by the dashed line in Figure 4 . After this point, the CL depins and evaporation continues under a combination of decreasing contact angle and radius, also known as mixed regime. On the roughest surface (S-6hr), however, the contact radius and the contact angle decrease continuously until a stickslip (SS) event happens at ca. $82 \%$ of its lifetime as the dashed line presented in Figure 4. The above different evaporation behaviours indicate potentially full wetting the first two cases, as described by Wenzel [10], and partial wetting in the roughest case, described by Cassie-Baxter [11]. 


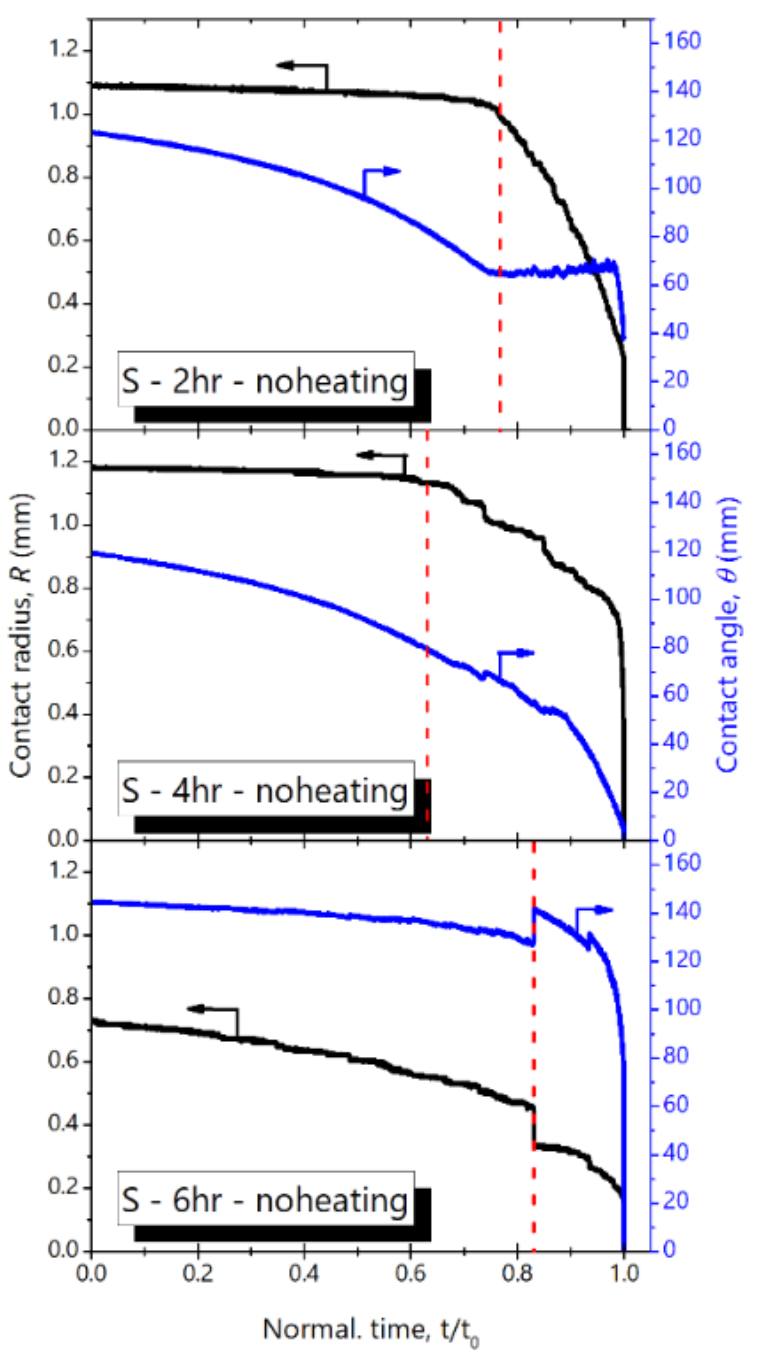

Figure 4. Evolution of the apparent contact angle, $\theta$, (blue line) and contact radius, $R$, (black line) as a function of normalized time, $\mathrm{t} / \mathrm{t}_{0}$, for pure water drops evaporating on non-heated surfaces with increasing roughness from top to bottom. Dashed lines show the moment of the transition of evaporation modes.

The evaporative behaviour of drops under localised heating differs from the nonheated ones. In Figure, we present the evolution over normalised time of the contact radius and the contact angle of water drops on hot spots with increasing roughness from top to bottom. On the smoother surface (S-2hr), the drop evaporates initially for ca. $80 \%$ of its lifetime, under the CCR regime with decreasing contact angle. Once the contact angle reaches a critical low value, the CL jumps to the next energetically favourable position similarly to SS evaporation, which can be observed as a simultaneous sharp 
increase in CA and decrease in $\mathrm{CR}[7,28,29]$. In the middle roughness case, ( $\mathrm{S}-4 \mathrm{hr}$ ), the drop exhibits a similar evaporative behaviour, albeit for a faster transition to SS regime at ca. $65 \%$ of drop lifetime. It is worth noting that in contrast to the cases of S$2 \mathrm{hr}$ and $\mathrm{S}-4 \mathrm{hr}$ hot-spots, no evident CL jumps can be found for non-heated drops evaporating on the same surfaces. This can be attributed to the fact that heating provides enough energy for the drops to overcome the pinning energy barrier. Nonetheless, further increasing surface roughness leads to a new evaporative behaviour. The drop on the roughest (S-6hr), evaporates until ca. $54 \%$ of lifetime under the CCA regime when a transition to SS evaporation occurs. In comparison to the non-heated S-6hr case, the number of CL jump increases when the drops evaporate on the S-6hr hot-spot. In what follows, we further probe CL motion behaviour directly above the drop, followed with an IR camera for increased solid-liquid contrast. 


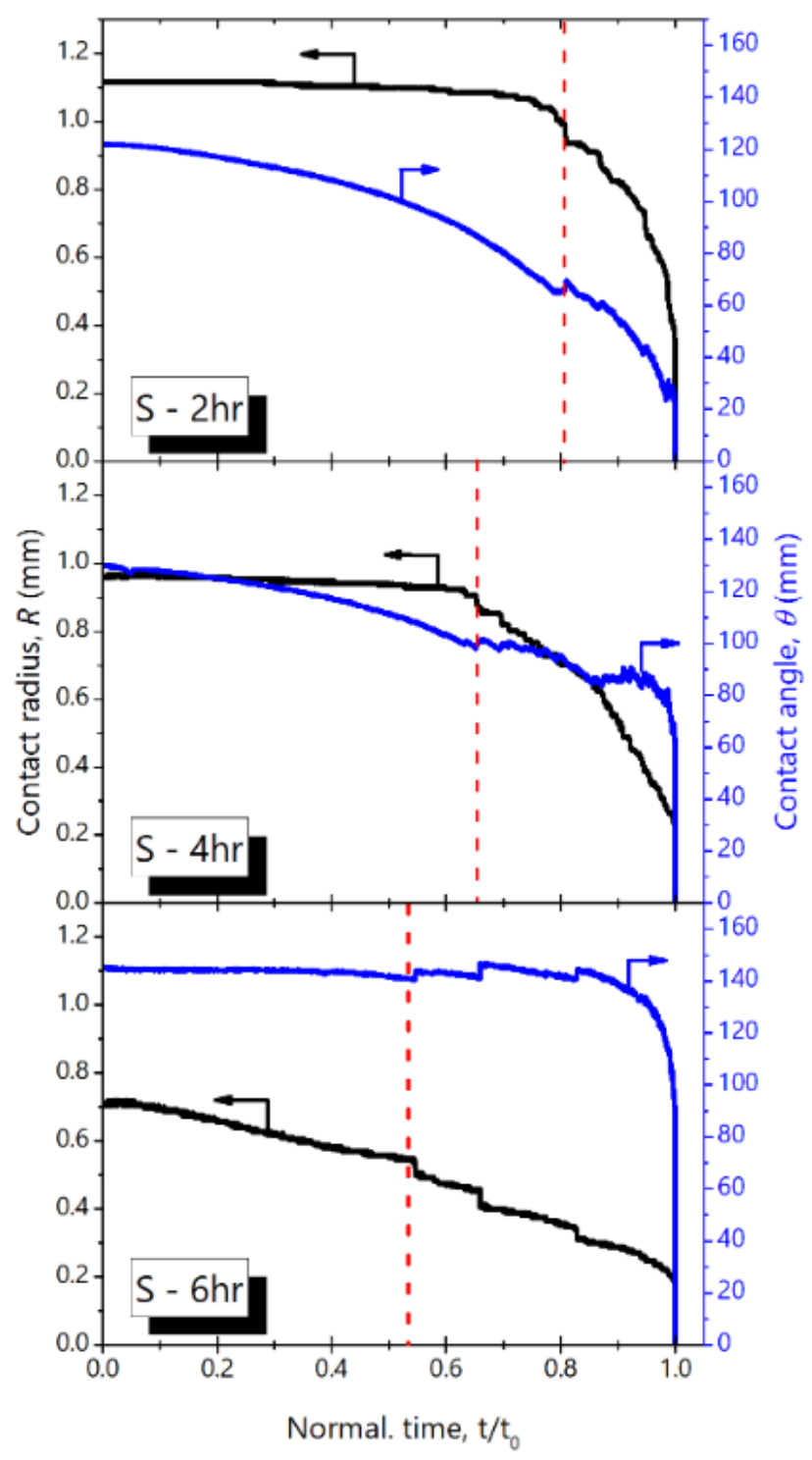

Figure 5. Evolution of the apparent contact angle, $\theta$, (blue line) and contact radius, $R$, (black line) as a function of normalized time, $\mathrm{t} / \mathrm{t}_{0}$, for pure water drops evaporating on hot-spots with increasing roughness from top to bottom. Dashed lines show the moment of the first jump.

\section{Contact line motion kinetics on hot-spots}

Figure 6 presents a schematic illustration of the top-view drop profiles over time for each hot-spot, captured with an IR camera for better liquid-solid contrast. The dashed lines represent the approximate profiles of CL after each rapid contraction or jump. Notably, the outer dashed line corresponds in each case to the initial stage of evaporation. Moreover, water is opaque to the spectrum of our IR camera and $\theta>90^{\circ}$, 
hence the actual CL should be slightly smaller.

In Figure 6 (a), it is readily apparent that the contact line in the $\mathrm{S}-2 \mathrm{hr}$ hot-spot is highly deformed by the surface asperities after each jump. In a similar, although weaker, fashion the CL of the drop evaporating on the $\mathrm{S}-4 \mathrm{hr}$ is deformed with a strong preferential pinning to one side, as shown in Figure 6 (b). Additionally, both drops exhibit a preferential one-sided pinning which is typical SS evaporation [30, 31]. On the other hand, the CL of drop evaporating on $\mathrm{S}-6 \mathrm{hr}$ appears to jump in a concentric fashion, indicating a weak solid-liquid interaction/pinning, as depicted in Figure 6 (c). The analysis above suggests that the deviation in evaporative behaviour between each surface presented above could be attributed to different wetting states. The drops on $\mathrm{S}$ $-2 \mathrm{hr}$ and $\mathrm{S}-4 \mathrm{hr}$ should completely wet the surfaces, as reported previously by Wenzel [7]. On the other hand, the rough asperities of the $S-6 \mathrm{hr}$ case allow air to be trapped between the solid and the liquid resulting in Cassie-Baxter or partial wetting [8]. In what follows, we will attempt to validate the wetting mode in each surface.

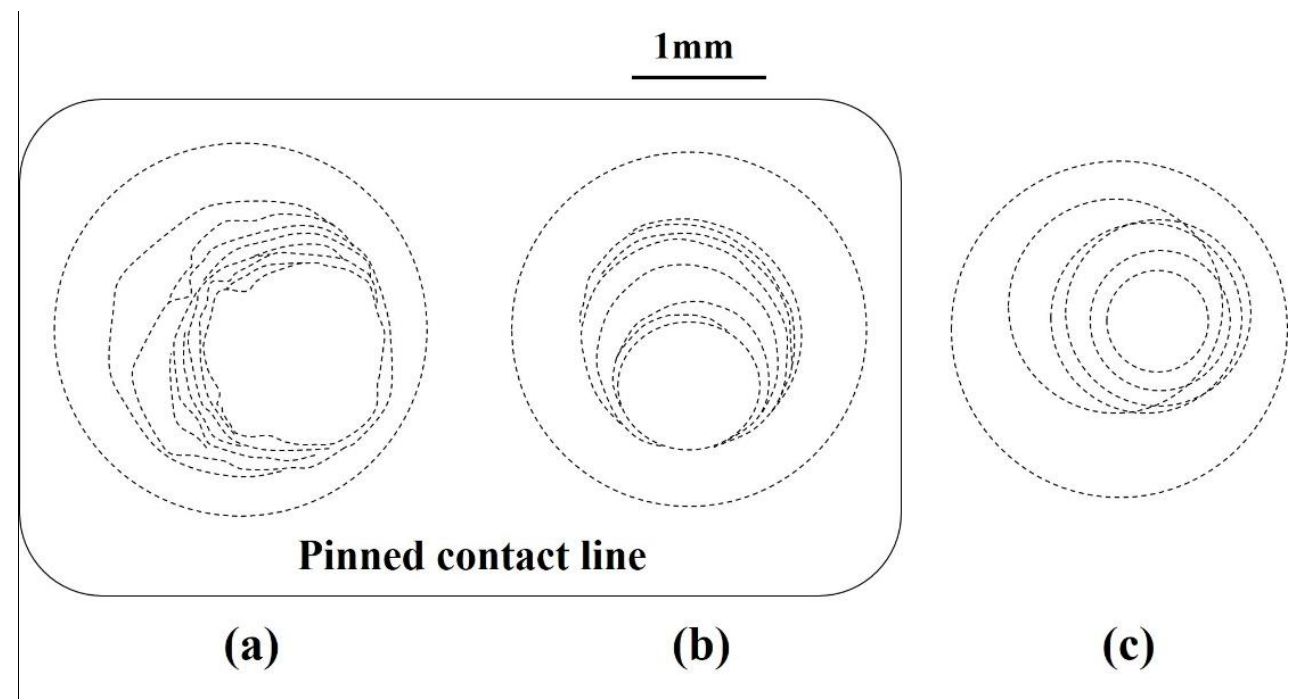

Figure 6. Schematic illustration of contact line location over time on (a) S-2hr, (b) S- 
4hr and (c) S-6hr after each jump.

Each contact line motion behaviour above can be categorized according to the wetting mode on each surface. The strong pinning behaviour on S-2hr and S-4hr hotspots is indicative of full wetting which we verified using the Wenzel criterion[7]: $\cos \theta_{a}^{W}=\mathrm{r} \cos \theta_{\mathrm{Y}}$, where $\theta_{a}^{W}$ is the Wenzel contact angle, $\theta_{Y} \sim 115^{\circ}$ is the Young's contact angle of a water drop on a smooth fluoro-resin surface. $r$ is defined as the ratio of the true surface area to its horizontal projection and was calculated as $r=1+$ $\mathrm{h} \sum_{i=1}^{N} \sqrt{A_{\text {pillar }}} / A$ with the height the surface microstructures, $h$, obtained from the CLSM images and $A_{\text {pillar, }}$ the area of the pillar tops obtained from the microscopy images.

On the other hand, the contact angle of S-6hr only obeys the Cassie-Baxter criterion given by[8]: $\cos \theta_{a}^{C B}=f\left(1+\cos \theta_{Y}\right)-1$, where $\theta_{a}^{C B}$ is the Cassie-Baxter contact angle and $f$ is the wetted solid fraction and is extracted from the microscopy images.

With knowledge of the wetting mode of each surface, we may now focus on the CL motion in each case. We plot in Error! Reference source not found. the total number of CL jumps observed from above, the average CL displacement and velocity as a function of roughness. Notably, we discern that each quantity decreases with increasing surface roughness. In other words, as the wetting state shifts from Wenzel to Cassie-Baxter the drop becomes more mobile, which results in a smaller number of CL jumps and smaller CL displacement/velocity. 

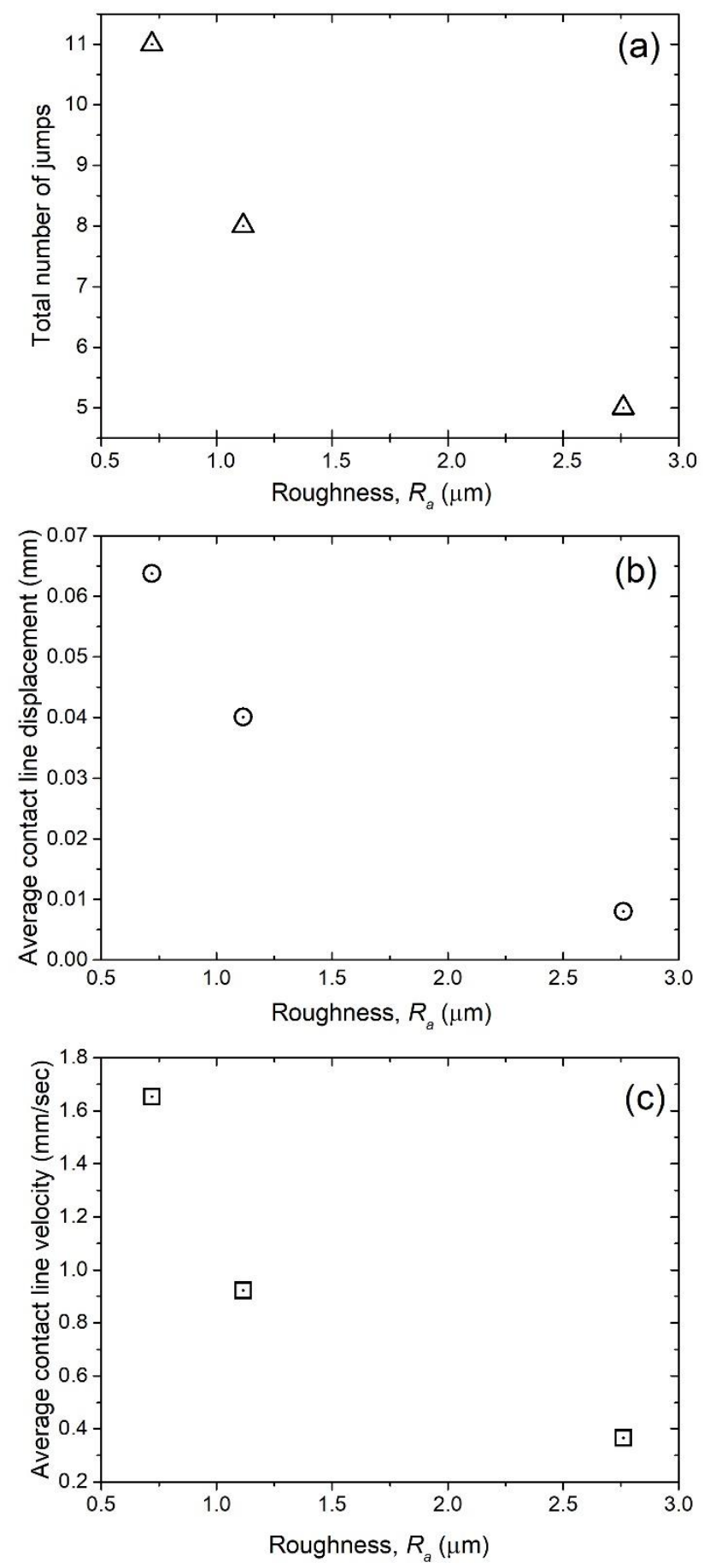

Figure 7. (a) Total number of jumps, (b) average displacement and (c) average velocity of contact line as a function of surface roughness for each hot-spot.

\section{Energy analysis}

In order to better understand the observed motion kinetics presented above, let us calculate the excess free energy of the drops on each surface, prior to the first CL jump. 
This energy should be roughly equal to the pinning energy barrier for the jump to ensue. We used the simple expression derived by Shanahan[28] which considers a sessile drop deviating from Young's equilibrium shape as it evaporates with pinned CL. Resultant change in the excess free energy, $\delta \bar{G}$, of the drop can then be written, in terms of $\theta$, as [32]: $\delta \bar{G}=\frac{\gamma_{l g} R(\delta \theta)^{2}}{2\left(2+\cos \theta_{a}\right)}$, where $\gamma_{l g}$ is the surface tension of water and $\delta \theta$ is the deviation in $\theta$ from the initial one.

Figure depicts the excess free energy for the first CL jump as a function of roughness for each hot-spot. It is readily apparent that the pinning barrier acting on the drops evaporating in fully wetting/Wenzel state is almost an order of magnitude higher than the partial wetting/Cassie-Baxter case. This fact could be interpreted by the smaller solid-liquid contact area in the CB case, leading to weaker adhesion. It is clear that due to the higher pinning barriers in Wenzel surfaces, the drops evaporate in CCR mode with pinned CL for a relatively longer time until the first jump as in Figure 5. Furthermore, a larger deviation from equilibrium due to a stronger pinning leads to greater and faster CL motion as shown in Figure 7[32].

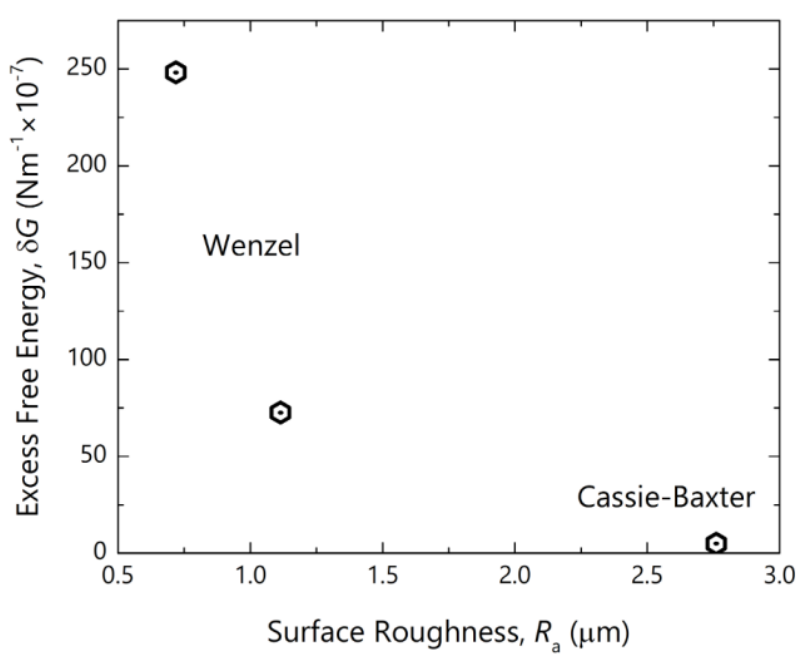

Figure 8. Exceess free energy as a function of roughness for each hot-spot. 


\section{Conclusion}

In this study, the evaporation process and the dynamics of contact line motion of drops on non-heated and locally heated surfaces, also known as hot-spots, with different degrees of roughness were studied. Drops evaporating on hot-spots were found to be more mobile due to the extra energy provided by heating. Roughness was found to result in either full or partial wetting. We validated numerically that the two smoother surfaces exhibited Wenzel or full wetting whereas the rougher one showed CassieBaxter or partial wetting. Derivation of the excess surface energy of each drop provides necessary insight into the actual pinning mechanism and interprets the observed drop motion behaviours. Our results will provide guidelines for the practical application of proposed novel cooling technologies for high-heat flux systems such as microelectronics. In these technologies, drops coalesce and jump (to minimise their energy) from the condenser surface to the hot-spot, where drops must remain mobile in order to be constantly replenished and hence remove more heat than pinned ones, which may grow large enough to act as thermal insulators.

\section{References}

[1] K. Ebrahimi, G. F. Jones, and A. S. Fleischer, "A review of data center cooling technology, operating conditions and the corresponding low-grade waste heat recovery opportunities," Renew. Sustain. Energy Rev., vol. 31, pp. 622-638, 2014. DOI: 10.1016/j.rser.2013.12.007.

[2] H. Zhang, S. Shao, H. Xu, H. Zou, and C. Tian, "Free cooling of data centers: A review," Renew. Sustain. Energy Rev., vol. 35, pp. 171-182, 2014. DOI: 10.1016/j.rser.2014.04.017.

[3] K. F. Wiedenheft et al., "Hotspot cooling with jumping-drop vapor chambers," Appl. Phys. Lett., vol. 110, no. 14, 2017. DOI: 10.1063/1.4979477. 
[4] J. Oh et al., "Jumping-droplet electronics hot-spot cooling," Appl. Phys. Lett., vol. 110, no. 12, pp. 1-6, 2017. DOI: 10.1063/1.4979034.

[5] M. C. Lopes and E. Bonaccurso, "Evaporation control of sessile water drops by soft viscoelastic surfaces," Soft Matter, vol. 8, pp. 7875-7881, 2012.

[6] D. Brutin and V. Starov, "Recent advances in droplet wetting and evaporation," Chem. Soc. Rev., vol. 47, pp. 558-585, 2018. DOI: 10.1039/c6cs00902f.

[7] Y. Kita et al., "Induction of Marangoni convection in pure water drops Induction of Marangoni convection in pure water drops," Appl. Phys. Lett., vol. 171602, 2016. DOI: 10.1063/1.4966542.

[8] G. McHale, S. Aqil, N. J. Shirtcliffe, M. I. Newton, and H. Y. Erbil, “Analysis of droplet evaporation on a superhydrophobic surface," Langmuir, vol. 21, no. 24, pp. 11053-11060, 2005. DOI: 10.1021/la0518795.

[9] P. B. Weisensee et al., "Droplet impact on vibrating superhydrophobic surfaces," Phys. Rev. Fluids, vol. 2, no. 10, pp. 1-14, 2017. DOI: 10.1103/PhysRevFluids.2.103601.

[10] R. N. Wenzel, "Resistance of solid surfaces to wetting by water," Ind. Eng. Chem., vol. 28, no. 8, pp. 988-994, 1936. DOI: 10.1021/ie50320a024.

[11] A. B. D. Cassie and S. Baxter, "Wettability of porous surfaces," Trans. Faraday Soc., vol. 40, no. 0, pp. 546-551, 1944. DOI: 10.1039/TF9444000546.

[12] J. M. Mabry and G. H. Mckinley, "Designing Superoleophobic Surfaces," no. August, pp. 1618-1623, 2008. DOI: 10.1126/science.1148326.

[13] C. Frankiewicz and D. Attinger, "Texture and wettability of metallic lotus leaves," Nanoscale, vol. 8, no. 7, pp. 3982-3990, 2016. DOI: 10.1039/C5NR04098A.

[14] Y. Y. Yan, N. Gao, and W. Barthlott, "Mimicking natural superhydrophobic surfaces and grasping the wetting process: A review on recent progress in preparing superhydrophobic surfaces," Adv. Colloid Interface Sci., vol. 169, no. 2, pp. 80-105, 2011. DOI: $10.1016 /$ j.cis.2011.08.005.

[15] H. Y. Erbil, "Evaporation of pure liquid sessile and spherical suspended drops: A review," Adv. Colloid Interface Sci., vol. 170, no. 1-2, pp. 67-86, 2012. DOI: 10.1016/j.cis.2011.12.006.

[16] W. Xu and C. H. Choi, "From sticky to slippery droplets: Dynamics of contact line depinning on superhydrophobic surfaces," Phys. Rev. Lett., vol. 109, pp. 1-5, 2012. DOI: 10.1103/PhysRevLett.109.024504.

[17] K.-H. Cho and L.-J. Chen, "Fabrication of sticky and slippery superhydrophobic surfaces via spin-coating silica nanoparticles onto flat/patterned substrates," Nanotechnology, vol. 22, p. 445706, 2011. DOI: 10.1088/0957-4484/22/44/445706.

[18] D. Bonn, J. Eggers, J. Indekeu, and J. Meunier, "Wetting and spreading," Rev. Mod. Phys., vol. 81, no. 2, 2009. DOI: 10.1103/RevModPhys.81.739.

[19] R. D. Deegan, O. Bakajin, T. F. Dupont, G. Huber, S. R. Nagel, and T. A. Witten, "Capillary flow as the cause of ring stains from dried liquid droplets," Nature, vol. 389, pp. 827-829, 1997. DOI: 10.1038/39827.

[20] K. Sefiane and L. Tadrist, "Experimental investigation of the de-pinning phenomenon on rough surfaces of volatile drops," Int. Commun. Heat Mass Transf., vol. 33, no. 4, 
pp. 482-490, 2006. DOI: 10.1016/j.icheatmasstransfer.2005.12.010.

[21] S. Dash and S. V. Garimella, "Droplet evaporation dynamics on a superhydrophobic surface with negligible hysteresis," Langmuir, vol. 29, no. 34, pp. 10785-10795, 2013. DOI: $10.1021 / \mathrm{la} 402784 \mathrm{c}$.

[22] F. Girard, M. Antoni, S. Faure, and A. Steinchen, "Influence of heating temperature and relative humidity in the evaporation of pinned droplets," Colloids Surfaces A Physicochem. Eng. Asp., vol. 323, no. 1-3, pp. 36-49, 2008. DOI: 10.1016/j.colsurfa.2007.12.022.

[23] S. Dash and S. V. Garimella, "Droplet evaporation on heated hydrophobic and superhydrophobic surfaces," Phys. Rev. E - Stat. Nonlinear, Soft Matter Phys., vol. 89, no. 4, pp. 1-8, 2014. DOI: 10.1103/PhysRevE.89.042402.

[24] M. Parsa, S. Harmand, K. Sefiane, M. Bigerelle, and R. Deltombe, "Effect of substrate temperature on pattern formation of nanoparticles from volatile drops," Langmuir, vol. 31, no. 11, pp. 3354-3367, 2015. DOI: 10.1021/acs.langmuir.5b00362.

[25] R. M. Do Nascimento, C. Cottin-Bizonne, C. Pirat, and S. M. M. Ramos, "Water Drop Evaporation on Mushroom-like Superhydrophobic Surfaces: Temperature Effects," Langmuir, vol. 32, no. 8, pp. 2005-2009, 2016. DOI: 10.1021/acs.langmuir.5b04445.

[26] C. Te Liu, W. H. Lee, and T. L. Shih, "Synthesis of $\mathrm{ZnO}$ nanoparticles to fabricate a mask-free thin-film transistor by inkjet printing," J. Nanotechnol., vol. 2012, 2012. DOI: $10.1155 / 2012 / 710908$.

[27] B. Qian and Z. Shen, "Fabrication of superhydrophobic surfaces by dislocationselective chemical etching on aluminum, copper, and zinc substrates," Langmuir, vol. 21, no. 20, pp. 9007-9009, 2005. DOI: 10.1021/la051308c.

[28] M. E. R. Shanahan, "Simple Theory of 'Stick-Slip' Wetting Hysteresis," Langmuir, vol. 11, no. 3, pp. 1041-1043, 1995. DOI: 10.1021/la00003a057.

[29] A. Askounis, Y. Takata, K. Sefiane, V. Koutsos, and M. E. R. Shanahan, “'biodrop' Evaporation and Ring-Stain Deposits: The Significance of DNA Length," Langmuir, vol. 32, no. 17, pp. 4361-4369, 2016. DOI: 10.1021/acs.langmuir.6b00038.

[30] D. Debuisson, A. Merlen, V. Senez, and S. Arscott, "Stick-Jump (SJ) Evaporation of Strongly Pinned Nanoliter Volume Sessile Water Droplets on Quick Drying, Micropatterned Surfaces," Langmuir, vol. 32, no. 11, pp. 2679-2686, 2016. DOI: 10.1021/acs.langmuir.6b00070.

[31] A. Askounis, D. Orejon, V. Koutsos, K. Sefiane, and M. E. R. Shanahan, "Nanoparticle deposits near the contact line of pinned volatile droplets: size and shape revealed by atomic force microscopy," Soft Matter, vol. 7, no. 9, p. 4152, 2011. DOI: $10.1039 / \mathrm{c} 1 \mathrm{sm} 05241 \mathrm{a}$

[32] D. Orejon, K. Sefiane, and M. E. R. Shanahan, "Stick-slip of evaporating droplets: Substrate hydrophobicity and nanoparticle concentration," Langmuir, vol. 27, pp. 12834-12843, 2011. DOI: 10.1021/la2026736 